\title{
T1-weighted Axial Visual Rating Scale for an Assessment of Medial Temporal Atrophy in Alzheimer's Disease
}

\author{
Geon Ha Kim ${ }^{\mathrm{a}}$, Jung-Eun Kim ${ }^{\mathrm{a}}$, Kyoung-Gyu Choi ${ }^{\mathrm{a}}$, Soo Mee Lim ${ }^{\mathrm{b}}$, Jong-Min Lee ${ }^{\mathrm{c}}$, Duk L. Na ${ }^{\mathrm{d}}$ \\ and Jee Hyang Jeong ${ }^{\mathrm{a}, *}$ \\ ${ }^{a}$ Department of Neurology, Ewha Womans University Mokdong Hospital, Ewha Womans University School of \\ Medicine, Seoul, Korea \\ ${ }^{\mathrm{b}}$ Department of Radiology, Ewha Womans University Mokdong Hospital, Ewha Womans University School of \\ Medicine, Seoul, Korea \\ ${ }^{\mathrm{c}}$ Department of Biomedical Engineering, Hanyang University, Seoul, Korea \\ ${ }^{\mathrm{d}}$ Department of Neurology, Samsung Medical Center, Sungkyunkwan University School of Medicine, Seoul, Korea
}

Accepted 15 January 2014

\begin{abstract}
.
Background: The most-widely used visual rating scale (VRS) for medial temporal atrophy is the T1-weighted (T1W) coronal VRS developed by Scheltens et al. However, it is often difficult to use the T1W-coronal VRS in cases with limitations in obtaining T1W-coronal images. To overcome this issue, we modified the T1W-coronal VRS onto the axial plane.

Objective: The purposes of this study were to validate our T1W-axial VRS by examining its compatibility with the original T1W-coronal VRS and by investigating the correlation with the cognitive functions and hippocampal volumes.

Methods: Participants were 50 patients with Alzheimer's disease dementia and 30 elderly with normal cognition. We transposed each component of the T1W-coronal VRS onto T1W-axial images (i.e., the largest height of the hippocampal formation into the width of the medial temporal lobe). The compatibility of T1W-axial VRS with T1W-coronal one was determined using the kappa value. The correlations of T1W-axial VRS with cognitive performance or the hippocampal volumes were analyzed with age, gender, and education as covariates.

Results: The kappa value between the T1W-axial and T1W-coronal VRS was $0.772(p<0.045)$. The T1W-axial VRS showed a significant correlation with the scores of cognitive functions, including verbal memory tests $(-0.601, p<0.001$ for the left). Furthermore, the T1W-axial VRS also correlated well with hippocampal volumes $(-0.576, p<0.001)$.

Conclusions: The T1W-axial VRS showed good agreement with T1W-coronal VRS and correlated well with cognitive functions as well as hippocampal volumes, which suggests that the T1-axial VRS may replace the original T1W-coronal one.
\end{abstract}

Keywords: Alzheimer's disease, medial temporal atrophy, visual rating scale

\footnotetext{
${ }^{*}$ Correspondence to: Jee Hyang Jeong, MD, PhD, Department of Neurology, Ewha Womans University Mokdong Hospital, Ewha Womans University School of Medicine [158-710], 1071 Anyangcheon-ro, Yangcheon-gu, Seoul, Korea. Tel.: +8222650 2776; Fax: +82 22650 2652; E-mail: jjeong@ewha.ac.kr.
}

\section{INTRODUCTION}

Alzheimer's disease (AD) is the most common cause of degenerative dementia among the elderly [1-3]. Memory impairment is the earliest and most prominent clinical manifestation, and medial temporal lobe atrophy (MTA) including the hippocampus is known to be the first neural correlate involved in $\mathrm{AD}$ [4-7]. 
Therefore, clinicians have evaluated MTA on magnetic resonance (MR) images since many studies have shown that the degree of MTA is a predictor of AD progression in patients with mild cognitive impairment [2, 6-10] and correlates well with the extent of cognitive impairment in patients with $\mathrm{AD}[8,11$, 12]. Recently, the National Institute on Aging and the Alzheimer's Association proposed revised criteria for $\mathrm{AD}$ where atrophy of the medial temporal lobe was included as a biomarker of neuronal damages [13]. As a consequence, the use of volumetric methods or visual assessments of MTA is expected to increase.

In many clinical studies, visual assessment of MTA has been used, and its advantages are that it is less time intensive and easier to apply than quantitative volumetric analysis. The most widely used visual rating scale (VRS) for MTA in clinical practice is the T1 weighted (T1W)-coronal VRS that was proposed by Scheltens et al. in 1992, in which the authors use T1Wcoronal MR images perpendicular to the brainstem $[5,7]$. This scale has shown meaningful and reliable results through many clinical studies [7, 14, 15].

However, it is not always easy to use the T1Wcoronal VRS for MTA by Scheltens et al. in cases with limited acquisition of T1W-coronal images. To overcome this issue, we modified the T1W-coronal VRS into an axial plane that allows the use of T1W-axial MR images for the visual rating of MTA. We examined the comparability between the T1W-axial and the T1Wcoronal VRS in our preliminary study, which showed that our T1W-axial VRS showed good agreement with T1W-coronal one [16].

The purpose of the current study was, therefore, to validate our modified T1W-axial VRS for MTA using the following methods. First, we aimed to demonstrate the compatibility of our new T1W-axial VRS with the original T1W-coronal VRS by Scheltens et al. in larger number of participants. Second, we investigated whether our T1W-axial VRS was correlated with the clinical severity. Lastly, we analyzed the correlation between our T1W-axial VRS and hippocampal volumes that were manually traced on the $\mathrm{T} 1 \mathrm{~W}$-coronal MR images.

\section{MATERIALS AND METHODS}

\section{Patients with $A D$ dementia}

A total of 27 patients were recruited from the Memory Disorder Clinic at Ewha Woman's University Mokdong Hospital and 23 patients from Samsung Medical Center in Seoul, Korea, from January 2007 to April 2008. All patients with AD dementia fulfilled the criteria for probable $\mathrm{AD}$ according to the National Institute of Neurological and Communicative Disorders and Stroke and the Alzheimer's Disease and Related Disorders Association [17]. All patients underwent clinical interviews and neurological examinations by an experienced neurologist (Dr. Jeong or Dr. Na) to evaluate their cognitive, behavioral, and functional impairments. We subsequently conducted detailed neuropsychological and laboratory tests including complete blood counts, blood chemistry, vitamin B12/folate, syphilis serology, and thyroid function tests to exclude secondary causes of cognitive defects. Patients who had cerebral infarction, hemorrhage, tumors, trauma, and severe white matter hyperintensity were excluded from the study population.

\section{Individuals with no cognitive impairment (NC)}

The 30 individuals who were spouses of the patients of the Samsung Medical Center were enrolled as controls for this study with their consent. They had no cognitive complaint, no neurological or psychiatric illnesses, and no abnormalities on neurological examinations. Their performances on the Korean version of Mini-Mental State Examination (K-MMSE) [18] and neuropsychological tests were within normal ranges.

\section{Standard protocol approvals, registrations, and patient consents}

The study was approved by the Institutional Review Board of Ewha Womans University Mokdong Hospital as well as Samsung Medical Center, and written informed consent was obtained from all participants enrolled in the study.

\section{Neuropsychological tests}

All participants received standardized neuropsychological tests using the Seoul Neuropsychological Screening Battery [19]. This battery comprised tests for attention, language, calculation, praxis, visuospatial/constructive function, verbal/visual memory, and frontal/executive function. The attention domain score was assessed by the forward and backward digit span test. The Korean version of the Boston Naming Test (K-BNT) and 12 trials of written calculations (three trials each for addition, subtraction, multiplication, and division) were included to test the performance of language and calculation. The copying test from the Rey 
Complex Figure Test (RCFT) was used to assess visuospatial function. The verbal memory was examined by the immediate recall, delayed recall task, and a recognition task from the Seoul Verbal Learning Test (SVLT). The visual memory was also assessed by the immediate recall, delayed recall, and recognition task of the RCFT. The frontal/executive function subdomain was assessed a word fluency and Stroop color-reading test.

\section{Visual rating of medial temporal atrophy}

Two neurologists (Dr. Jeong and Dr. Kim) rated MTA grading independently after a series of training sessions. The scans were numbered to hide the patients' clinical information and were gathered separately in $\mathrm{T} 1 \mathrm{~W}$-coronal and $\mathrm{T} 1 \mathrm{~W}$-axial images for visual rating. The raters judged all scans in a random order, which was different for each rater. T1W-coronal and $\mathrm{T} 1 \mathrm{~W}$-axial images were analyzed separately. Interrater reliability between the two raters and intra-rater agreement were obtained with a seven-day interval between the two ratings. For the correlation analyses of VRS with cognitive function, clinical severity and hippocampal volumes, we used only the scores of VRS at the first trial of the rater 1. Especially in the analysis of diagnostic performance, the more severe MTA was selected for an each participant if the degree of MTA was asymmetric between the left and right.

\section{T1W-coronal visual rating scale for MTA by Scheltens et al.}

On the T1W-coronal VRS, the degree of MTA is rated on 5-point scale from absent (0) to severe (4) [7]. The three linear measures of the medial temporal lobe (A, C, and D) were calculated for both sides (left and right). $\mathrm{A}$ is the largest height of the hippocampal formation defined as the dentate gyrus, hippocampus proper, and subiculum together with the parahippocampal gyrus. $\mathrm{C}$ is the vertical width of the choroid fissure centered on the midpoint of the hippocampus, and $\mathrm{D}$ is the width of the temporal horn (TH) (Fig. 1A).

\section{T1W-axial visual rating scale for MTA}

We transposed the T1W-coronal VRS [7] onto the T1W-axial images (Fig. 1B, Table 1). The greatest height of the hippocampal formation (A) on the T1Wcoronal scale was converted to the width of the medial temporal lobe (A') on the $\mathrm{T} 1 \mathrm{~W}$-axial scale, while the vertical width of the choroid fissure (C) was super- seded by the perimesencephalic cistern (PC) gap (C') measured by the width between the brainstem and the medial temporal lobe. Finally, the width of the TH (D) was substituted by the width of the anterior TH of the lateral ventricle (D').

The defined measurements for the axial VRS are as follows (Fig. 1, Table 1): $A^{\prime}$ is the width of medial temporal lobe (comprising the hippocampusparahippocampal gyrus); C' represents the PC gap (the width of the crural cistern); and D' is the width of anterior TH. The axial-MTA scale ranged from 0 (no atrophy) to 4 (severe atrophy): Grade 0, no MTA change without widening of the $\mathrm{PC} / \mathrm{TH}$ and a normal medial temporal cortex; Grade 1, questionable atrophy of the medial temporal lobe with slight widening of the perimesencephalic cistern/or slit-like TH; Grade 2, mild but definite change of the MTA showing mild widening of the PC combined with mild but definite widening of the $\mathrm{TH}$; Grade 3 , moderate change of the MTA and moderate widening of PC/TH with bending of the hippocampus proper; and Grade 4, severe change of the MTA showing severe widening of the $\mathrm{PC} / \mathrm{TH}$ with marked angulation by knife-edge change of the medial temporal cortex (Fig. 1, Table 1).

\section{MR image acquisition}

Twenty-seven patients with AD dementia were referred to Ewha Womans University Mokdong Hospital for MR images taken with a 1.5-T MRI scanner (Avanto Syngo, Seimens, Erlangen, Germany) using a 12 channel head coil, whereas 23 patients with AD dementia and 30 with $\mathrm{NC}$ underwent brain MRI using a 1.5-T MRI scanner (Signa, GE, Milwaukee, WI, USA) with a head coil at Samsung Medical Center. The MR images were acquired employing five different techniques; T1W-3D magnetization-prepared rapid gradient echo (MPR) coronal for Seimens or 3D spoiled gradient echo (SPGR) coronal for GE Signa, T1W- axial, FLAIR axial, T2W axial, and GRE. All images were acquired using identical imaging protocols and the same slice positions as follows. The $\mathrm{T} 1 \mathrm{~W}$-coronal images were obtained with following parameters; slice thickness, $1.2 \mathrm{~mm}$; inter slice thickness of $0 \mathrm{~mm}$; repetition time (TR): $1160 \mathrm{~ms}$; echo time (TE): $4.19 \mathrm{~ms}$, flip angle: $15^{\circ}$, matrix size of $256 \times 192$ and field of view (FOV) $188 \times 250 \mathrm{~mm}$. The T1Waxial images that were parallel to the AC-PC line, which was performed with $5.0 \mathrm{~mm}$ slice thickness, inter-slice thickness, $1.5 \mathrm{~mm}$, TR: $550 \mathrm{~ms}$, TE: $17 \mathrm{~ms}$, and flip angle: $90^{\circ}$, matrix size of $256 \times 190$ pixels and FOV $1750 \times 200 \mathrm{~mm}$. 
(A) T1W-coronal visual rating scale
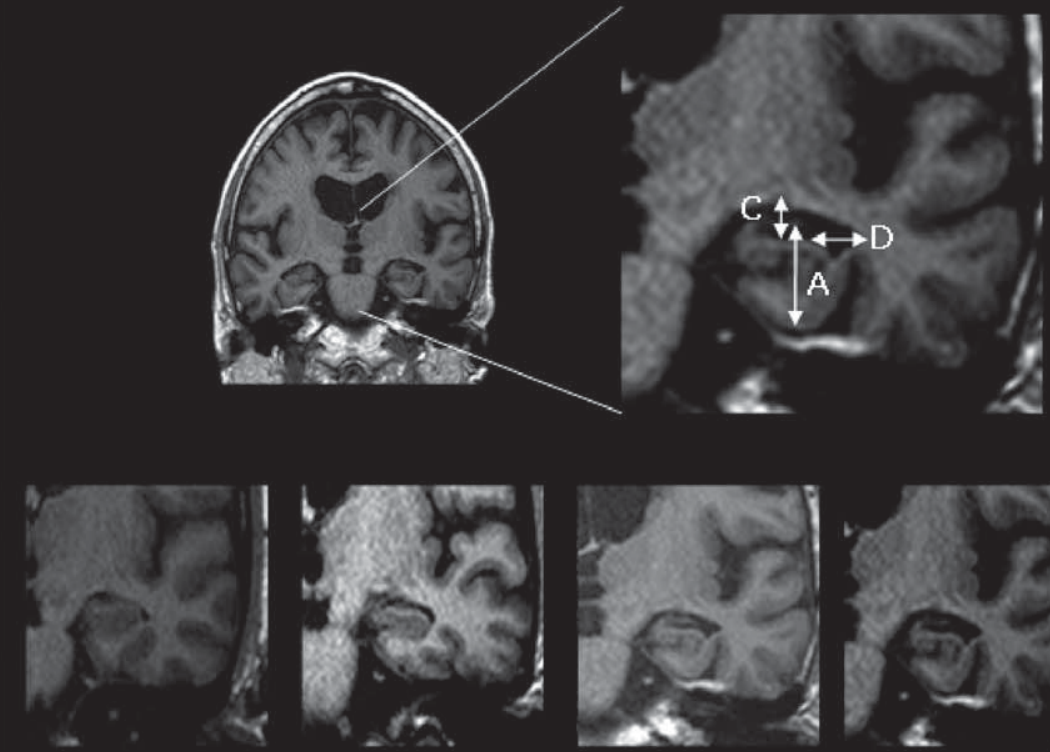

Grade 0

Grade 1

Grade 2

Grade 3

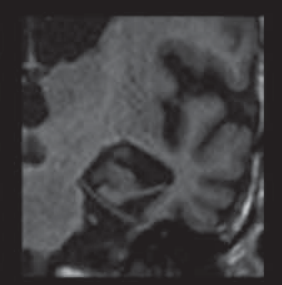

Grade 4

(B) T1W-axial visual rating scale

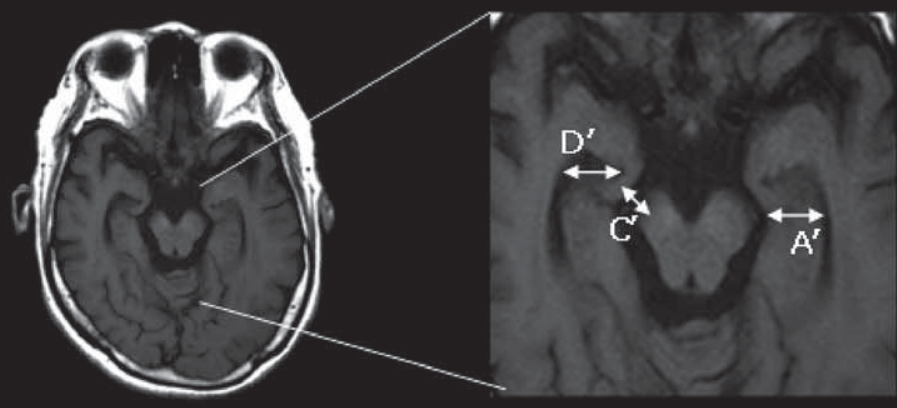

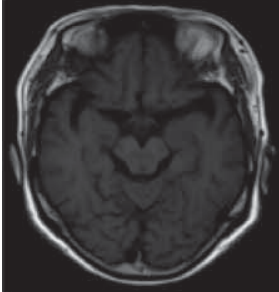

Grade 0

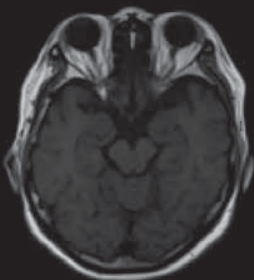

Grade 1

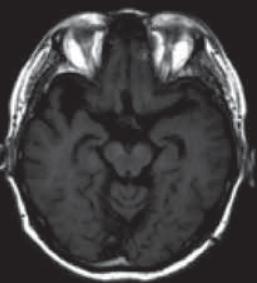

Grade 2

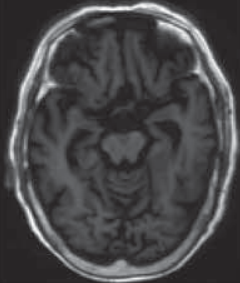

Grade 3

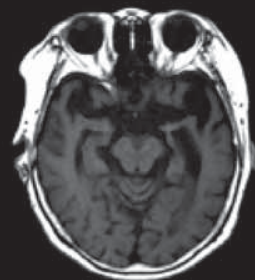

Grade 4 (Left)

Fig. 1. T1W-axial visual rating scale. (A) T1W-coronal visual rating scale. A) Height of hippocampal formation, C) Vertical width of the choroid fissure, D) Width of the temporal horn. (B) T1W-coronal visual rating scale. A') Width of the hippocampus, C') Width of the perimesencephalic cistern, D') Width of the temporal horn. 
Table 1

Comparison of the T1W-axial visual rating scale with the T1W-coronal visual rating scale for MTA

\begin{tabular}{|c|c|c|c|c|c|c|}
\hline & \multicolumn{3}{|c|}{ T1W-coronal visual rating scale } & \multicolumn{3}{|c|}{ T1W-axial visual rating scale } \\
\hline & $\begin{array}{l}\text { A: Height of } \\
\text { hippocampal } \\
\text { formation }\end{array}$ & $\begin{array}{c}\text { C: Vertical } \\
\text { width of the } \\
\text { choroid fissure }\end{array}$ & $\begin{array}{l}\text { D: Width of } \\
\text { the temporal } \\
\text { horn }\end{array}$ & $\begin{array}{l}\text { A': Medial } \\
\text { temporal } \\
\text { lobe }\end{array}$ & $\begin{array}{c}C^{\prime}: \text { Perimesencephalic } \\
\text { cisternal gap }\end{array}$ & $\begin{array}{c}\text { D': Width of } \\
\text { anterior temporal } \\
\text { horn }\end{array}$ \\
\hline Grade 0: Normal & $\mathrm{N}$ & $\mathrm{N}$ & $\mathrm{N}$ & $\mathrm{N}$ & $\mathrm{N}$ & $\mathrm{N}$ \\
\hline Grade 1: Questionable & $\mathrm{N}$ & $\uparrow$ & $\mathrm{N}$ & $\mathrm{N}$ & $\uparrow$ & $\mathrm{N}$ or slit-like change \\
\hline Grade 2: Mild & $\downarrow$ & $\uparrow \uparrow$ & $\uparrow$ & $\downarrow$ & $\uparrow \uparrow$ & $\uparrow$ \\
\hline Grade 3: Moderate & $\downarrow \downarrow$ & $\uparrow \uparrow \uparrow$ & $\uparrow \uparrow$ & $\downarrow \downarrow$ & $\uparrow \uparrow \uparrow$ & $\uparrow \uparrow$ \\
\hline Grade 4: Severe & $\downarrow \downarrow \downarrow$ & $\uparrow \uparrow \uparrow$ & $\uparrow \uparrow \uparrow$ & $\downarrow \downarrow \downarrow$ & $\uparrow \uparrow \uparrow$ & $\uparrow \uparrow \uparrow$ \\
\hline
\end{tabular}

$\uparrow$, widening or increasing in size; $\downarrow$, decrease; n, normal.

\section{Hippocampal volumetric analyses}

The hippocampal volumetric analyses were performed on all the participants by a single specialist who was blinded to the participant's details at the time of the assessment. Manual tracing of the hippocampus on T1W-coronal MR images was assessed with Analyze 8.1 (Mayo Clinic, Rochester, MN, USA). Each image was binarized into the region of interest (ROI) and the rest. The ROI part was regarded as ' 1 ', and the rest as ' 0 '. We counted the number of voxels in the delineated area on each slice and then multiplied the total imaging voxels by the voxel volume $\left(0.5 \times 0.5 \times 1.2 \mathrm{~mm}^{3}\right)$. Anatomic guidelines for the structures of interest were established using Duvernoy's anatomic sections [20]. We selected 10 participants randomly and repeated the examination for hippocampal volume after 14 days to examine the intra-rater reliability of volumetry, which showed a high correlation coefficient value (intra-rater correlation coefficient: 0.996 , $p<0.001)$.

\section{Statistical analyses}

Statistical analyses were performed using the Statistical Package for the Social Sciences 18.0 (SPSS Inc., Chicago, IL, USA). Descriptive statistics of the initial work up were performed using demographic and clinical scores. Student's $t$-test or the Mann-Whitney $\mathrm{U}$ test was used to assess continuous variables, and the chi-square test was used to assess dichotomous variables. The compatibility of the T1W-axial VRS with T1W-coronal one was examined using the kappa value, which was also employed for the inter-rater and intra-rater agreement of the $\mathrm{T} 1 \mathrm{~W}$-axial and $\mathrm{T} 1 \mathrm{~W}$ coronal VRS. The analysis of covariance (ANCOVA) was used to compare the mean hippocampal volumes between the patients with AD and NC after adjusting for age, gender, and education as covariates. The correlations of T1W-axial VRS with cognitive performance or the hippocampal volumes were analyzed with the same covariates. The diagnostic performance to discriminate $\mathrm{AD}$ from $\mathrm{NC}$ in the two VRS was evaluated using the area under the curve (AUC) value that was obtained from the Receiver Operating Characteristic (ROC) curve analysis [21]. The cut-off values of each VRS were obtained by the highest Youden's index methods [22, 23].

The statistical significance was defined as $p<0.05$.

\section{RESULTS}

\section{Demographic characteristics of participants}

The patients with AD dementia were older and less educated than the NC group $(p<0.001)$. The scores of VRS for MTA were significantly higher in AD patients compared to NC participants $(p<0.001)$. More detailed demographic characteristics are presented in Table 2.

\section{Comparison of the kappa value between the $T 1 W$-coronal and T1W-axial visual rating scale}

The kappa value for comparability between the T1W-coronal and T1W-axial VRSs was 0.772 ( $p<0.042,95 \%$ CI: 0.692-0.852). The inter-rater reliability of the T1-coronal VRS was $0.628(p<0.001)$, while that of the T1W-axial VRS was $0.636(p<0.001)$, which showed no significant difference of inter-rater reliability between the $\mathrm{T} 1 \mathrm{~W}$-coronal and $\mathrm{T} 1$-axial VRS (Supplementary Table 1). Intra-rater reliability of the T1W-axial VRS seemed to be higher than that of the T1W-coronal VRS (Rater 1:0.946, Rater 2:0.622 in the T1W-axial VRS versus Rater 1:0.835, Rater 2:0.529 in the T1W-coronal VRS), although the significance was negligible (Supplementary Table 1). 
Table 2

Demographic characteristics of the participants

\begin{tabular}{|c|c|c|c|}
\hline & $\begin{array}{l}\text { AD dementia } \\
\quad(n=50)\end{array}$ & $\begin{array}{l}\text { Normal cognitive individuals } \\
\qquad(n=30)\end{array}$ & $\overline{p \text {-value (Adjusted } p \text { ) }}$ \\
\hline Age (years) & $72.2 \pm 7.9$ & $66.2 \pm 6.8$ & $<0.001$ \\
\hline Gender (M:F) & $9: 41$ & $8: 22$ & 0.359 \\
\hline Education (years) & $7.3 \pm 4.7$ & $12.5 \pm 4.3$ & $<0.001$ \\
\hline K-MMSE & $21.1 \pm 5.4$ & $28.6 \pm 1.2$ & $<0.001 *(<0.001 *)$ \\
\hline CDR & $0.8 \pm 0.6$ & $0.4 \pm 0.1$ & $0.017 *(0.024 *)$ \\
\hline CDR (sum of box) & $4.4 \pm 3.7$ & $0.6 \pm 0.3$ & $<0.001(0.001 *)$ \\
\hline \multicolumn{4}{|c|}{ MTA visual rating scale Coronal visual rating } \\
\hline Right & $2.1 \pm 0.9$ & $0.7 \pm 0.6$ & $<0.001 *(<0.001 *)$ \\
\hline Left & $2.0 \pm 0.8$ & $0.8 \pm 0.6$ & $<0.001 *(0.003 *)$ \\
\hline \multicolumn{4}{|l|}{ Axial visual rating } \\
\hline Right & $2.1 \pm 0.9$ & $0.9 \pm 0.7$ & $0.002 *(0.002 *)$ \\
\hline Left & $2.1 \pm 0.8$ & $0.9 \pm 0.7$ & $<0.001 *(0.010 *)$ \\
\hline \multicolumn{4}{|c|}{ Hippocampal volume $\left(\mathrm{mm}^{3}\right)$} \\
\hline Right & $1489.3 \pm 759.4$ & $3272.9 \pm 1482.3$ & $<0.001 *(<0.001 *)$ \\
\hline Left & $1393.3 \pm 711.3$ & $3116.3 \pm 1476.2$ & $<0.001 *(<0.001 *)$ \\
\hline
\end{tabular}

\section{Correlation between the $T 1 W$-axial visual rating scale and the clinical and cognitive severity}

Our T1W-axial VRS correlated well with the clinical severity measured by the scores of Clinical Dementia Rating (CDR) $(\rho=0.317$ for the right, 0.379 for the left) and the CDR sum of boxes ( $\rho=0.357$ for the right, 0.430 for the left). In addition, significant correlation was also evident between the T1W-axial VRS and general cognitive function, which was assessed by the K-MMSE. The correlation coefficient between T1Waxial VRS and K-MMSE scores was -0.313 in the right MTA $(p=0.036)$ versus -0.404 for the left MTA $(p=0.006)$. The T1W-axial VRS also showed good correlation with the immediate $(-0.363$ for the right, -0.489 for the left), delayed recall scores $(-0.488$ for the right, -0.601 for the left) as well as recognition scores $(-0.295$ for the right, -0.456 for the left) of SVLT. Furthermore, the T1W-axial VRS correlated well with the scores of RCFT and several frontal executive functions including COWAT animals and color reading of Stroop tasks. Details are provided in Table 3.

Correlation between the $T 1 W$-axial visual rating scale and the hippocampal volume

There was significant correlation between the T1Waxial VRS and hippocampal volumes. The correlation coefficient was -0.613 for the right $(p<0.001)$ and -0.609 for the left $(p<0.001)$. The overall correlation of T1W-axial VRS and hippocampal volume was also high at -0.606 ( $p<0.001$, Fig. 2).

\section{Diagnostic accuracy of T1W-axial VRS and T1W-coronal VRS}

When the cut-off $\geq 2$ was defined as an indicator of the existence of hippocampal atrophy, the AUC value of T1W-axial VRS was 0.780 (95\% CI: 0.672-0.888) with a sensitivity $76.0 \%$, specificity of $80.0 \%$, and an accuracy of $77.5 \%$, which suggest fairly good performance for discriminating AD dementia from NC. In the T1W-coronal VRS, the AUC value was 0.787 (95\% CI: $0.681-0.892)$ with a sensitivity $74.0 \%$, specificity $83.3 \%$, and an accuracy of $77.6 \%$. There was no significant difference in the AUC values between the two VRS (95\% CI: $0.672-0.888$ in the T1W-axial VRS versus 95\% CI: $0.681-0.892$ in the T1W-coronal VRS).

\section{DISCUSSION}

The purpose of this study was to validate the modified T1W-axial VRS for MTA using a compatibility assessment with T1W-coronal VRS [7] and using correlation analyses with clinical severity, cognitive dysfunction, and hippocampal volumes.

First, we found a high kappa value between the T1W-axial and T1W-coronal VRSs, which suggests that our T1W-axial VRS was comparable with the T1W-coronal VRS $(0.772, p<0.045)$. Second, there was a significant correlation between the $\mathrm{T} 1 \mathrm{~W}$-axial VRS and the scores of the CDR or the CDR sum of boxes, which indicates that the T1W-axial VRS corresponded well with the clinical severity of the participants. Third, the current study showed that the 
Table 3

Correlation of the T1W-axial visual rating scale with clinical severity and cognitive function

\begin{tabular}{|c|c|c|c|c|c|c|c|c|}
\hline & \multicolumn{4}{|c|}{ T1W-Coronal visual rating scale } & \multicolumn{4}{|c|}{ T1W-Axial visual rating scale } \\
\hline & Right MTA & $p$-value & Left MTA & $p$-value & Right MTA & $p$-value & Left MTA & $p$-value \\
\hline \multicolumn{9}{|l|}{ Attention } \\
\hline Digit span: forward & 0.262 & 0.082 & 0.191 & 0.210 & 0.223 & 0.142 & 0.166 & 0.276 \\
\hline Digit span: backward & -0.155 & 0.311 & -0.181 & 0.235 & -0.118 & 0.442 & -0.199 & 0.189 \\
\hline \multicolumn{9}{|l|}{ Language } \\
\hline K-BNT & -0.269 & 0.074 & -0.344 & $0.021 *$ & -0.219 & 0.149 & -0.226 & 0.135 \\
\hline \multicolumn{9}{|l|}{ Visuospatial function } \\
\hline RCFT & -0.356 & $0.016^{*}$ & -0.204 & 0.179 & -0.292 & $0.001 *$ & -0.225 & 0.137 \\
\hline \multicolumn{9}{|l|}{ Memory } \\
\hline SVLT: immediate recall & -0.452 & $0.002 *$ & -0.507 & $<0.001 *$ & -0.363 & $0.014 *$ & -0.489 & $0.001 *$ \\
\hline SVLT: delayed recall & -0.532 & $<0.001 *$ & -0.545 & $<0.001 *$ & -0.488 & $0.001 *$ & -0.601 & $<0.001 *$ \\
\hline SVLT: recognition & -0.341 & $0.022 *$ & -0.391 & $0.008 *$ & -0.295 & $0.049^{*}$ & -0.456 & $0.002 *$ \\
\hline RCFT: immediate recall & -0.417 & $0.004 *$ & -0.483 & $0.001 *$ & -0.321 & $0.032 *$ & -0.392 & $0.008^{*}$ \\
\hline RCFT: delayed recall & -0.448 & $0.002 *$ & -0.492 & $0.001^{*}$ & -0.359 & $0.015^{*}$ & -0.404 & $0.006^{*}$ \\
\hline RCFT: recognition & -0.452 & $0.002 *$ & -0.475 & $0.001^{*}$ & -0.463 & $0.001 *$ & -0.527 & $<0.001 *$ \\
\hline \multicolumn{9}{|l|}{ Frontal/executive function } \\
\hline COWAT: animals & -0.410 & $0.005^{*}$ & -0.497 & $0.001 *$ & -0.326 & $0.029 *$ & -0.409 & $0.005^{*}$ \\
\hline COWAT: supermarket & -0.365 & $0.014 *$ & -0.442 & $0.002 *$ & -0.275 & 0.068 & -0.382 & $0.010 *$ \\
\hline COWAT: phonemic & -0.268 & 0.075 & -0.391 & $0.006^{*}$ & -0.167 & 0.273 & -0.307 & $0.041 *$ \\
\hline Stroop test: word & -0.233 & 0.123 & -0.29 & 0.053 & -0.234 & 0.121 & -0.205 & 0.177 \\
\hline Stroop test: color & -0.402 & $0.006^{*}$ & -0.517 & $<0.001^{*}$ & -0.308 & $0.040 *$ & -0.369 & $0.013 *$ \\
\hline K-MMSE & -0.362 & $0.015^{*}$ & -0.396 & $0.007 *$ & -0.313 & $0.036^{*}$ & -0.404 & 0.006 \\
\hline CDR & 0.345 & $0.020 *$ & 0.350 & $0.018^{*}$ & 0.317 & $0.034 *$ & 0.379 & $0.010 *$ \\
\hline CDR_sum of boxes & 0.384 & $0.009 *$ & 0.441 & $0.002 *$ & 0.357 & $0.016^{*}$ & 0.430 & $0.003 *$ \\
\hline
\end{tabular}

$* p<0.05$ partial correlation coefficient adjusted by age, gender and education SVLT, Seoul Verbal Learning Test; RCFT, Rey-Osterrieth Complex Figure Test; COWAT, Controlled Oral Word Association Test; K-MMSE, Korean version of Mini-Mental Status Examination; CDR, Clinical Dementia Rating.

T1W-axial VRS correlated with the scores of cognitive function, including K-MMSE, SVLT, and RCFT, which suggests the T1W-axial VRS matched well with general cognitive function as well as visual and verbal memory functions. Finally, we found that there was a significant correlation between the T1W-axial VRS and the hippocampal volumes, which suggests that our T1W-axial VRS reflected the hippocampal volumes of the brain.

In the modified $\mathrm{T} 1 \mathrm{~W}$-axial VRS, we assessed the entire medial side of the temporal lobe, including the hippocampus and the parahippocampal gyrus (A'), since it is difficult to delineate the hippocampus alone in the axial images. The T1W-coronal VRS also evaluates the hippocampal formation that encompasses the hippocampus proper, subiculum, and the parahippocampal gyrus together, and this measurement was similar to our T1W-axial VRS. The vertical width of the choroidal fissure (C) in the T1W-coronal scale was converted to the width of the PC in the T1W-axial VRS since the choroidal fissure is connected to the ambient cistern through the transverse fissure of Bichat, which is an inferior-medial extension of the choroidal fissure [24]. In addition, previous studies have shown that the enlargement of cerebrospinal fluid spaces in the perihippocampal region is indicative of atrophy and volume losses in the hippocampus and the parahippocampal gyrus [24, 25]; therefore, we included the width of the PC as a variable in our T1W-axial VRS.

The first major finding of this study was that the proposed modified T1W-axial VRS was well correlated with the well-known T1W-coronal VRS by Scheltens et al. (kappa value $=0.772 ; p<0.042$ ). The high kappa value between the $\mathrm{T} 1 \mathrm{~W}$-coronal and $\mathrm{T} 1 \mathrm{~W}$-axial VRS revealed that our T1W-axial VRS was compatible to the T1W-coronal VRS. In addition, the inter-rater and intra-rater reliability of the T1W-axial VRS seemed to be higher than the T1W-coronal VRS, although the significance was not considerable. It is also noteworthy that there was no difference of the diagnostic performance for discriminating $\mathrm{AD}$ from $\mathrm{NC}$ between the T1W-coronal and the axial VRS (AUC: 0.787 in the coronal versus 0.780 in the axial VRS) This high compatibility of T1W-axial VRS with the T1W-coronal one may provide an evidence for a reliable application of our T1W-axial VRS in clinical practice.

The second major finding in this study was that our T1W-axial VRS was correlated well not only with cognitive function but also with clinical severity. Table 3 demonstrates that our T1W-axial VRS was correlated with visual and verbal memory as well as global cognitive function measured by K-MMSE. However, the 

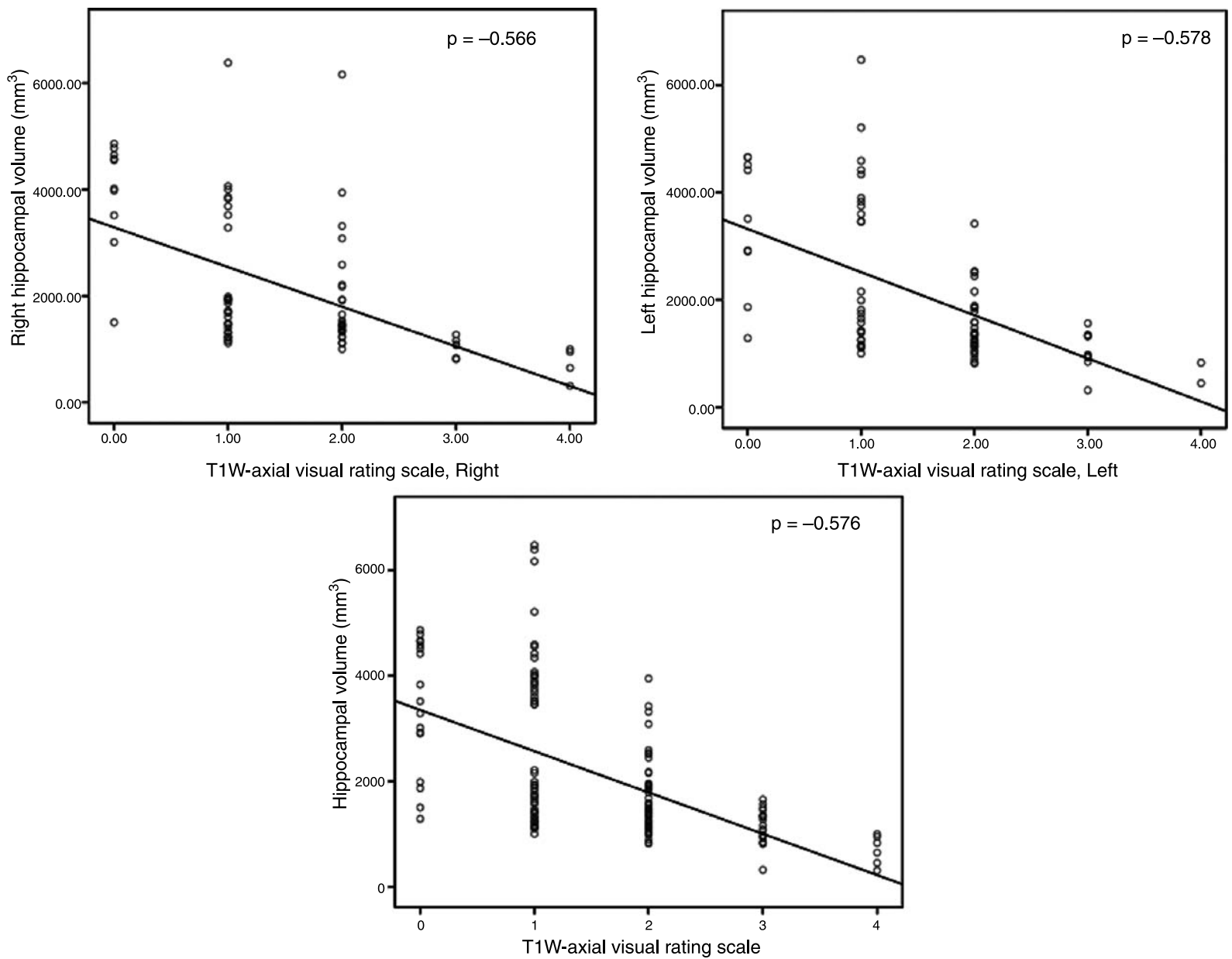

Fig. 2. Correlation between the T1W-axial visual rating and hippocampal volume. There was significant correlation between the T1W-axial visual rating scale and the hippocampal volumes. The correlation coefficient was -0.566 for the right $(p<0.001)$ and -0.578 for the left $(p<0.001)$. The overall correlation of the T1W-axial visual rating scale and the hippocampal volume was also fairly good at $-0.576(p<0.001)$. $\rho=$ Spearman's rho adjusted by age, gender, and education.

scores of other cognitive functions, such as attention and language functions, were not correlated with our T1W-axial VRS. It is noteworthy that our T1W-axial VRS for MTA was specifically correlated with visual and verbal memory function, which are known to be important functions mediated by the hippocampus [26]. This link might also indicate the potency of VRS for MTA in clinical practice.

The third important finding in this study was that the T1W-axial VRS also reflected the degree of hippocampal atrophy. The assessment of MTA has been a routine investigation, especially in dementia clinics, since MTA has been found to be an early and sensitive marker for $\mathrm{AD}$ [2]. Therefore, several techniques have been promoted to assess MTA, including volumetric measurement, linear measurement, or visual rating scales. For routine clinical use, however, the vol- umetric measurements have several drawbacks, such as stringent requirements of image quality and acquisition techniques, although they may provide truly quantitative measures of regional brain volumes. Considering the high correlation coefficient between the T1W-axial VRS and the hippocampal volume in our study, the T1W-axial VRS may be applied to routine dementia investigations as an alternative for volumetric measurement.

There were some limitations to our study. First, our normal controls were not age- and educationmatched participants, though we adjusted these factors as covariates when we compared the scores of VRS or cognitive tests. Second, we did not examine whether the T1W-axial VRS would correlate with hippocampal volumes after adjusting the total intracranial volumes. Therefore, it is possible that the modified T1W-axial 
VRS for MTA reflects global brain atrophy rather than a distinctive and disproportionate loss of hippocampal tissue relative to other brain regions.

Nonetheless, the modified T1W-axial VRS for MTA showed high comparability with the previous T1Wcoronal VRS with similar intra-rater and inter-rater reliability, which suggests the proposed T1W-axial VRS could replace the T1W-coronal VRS when there are limitations in obtaining $\mathrm{T} 1 \mathrm{~W}$-coronal images. Although not formally evaluated in the current study, we could expect that our T1W-axial VRS would be applied to CT images considering of the previous study showing that using a 64-detector row CT could obtain comparable information with MR imaging [27]. However, further studies with CT images should be needed to apply our T1W-axial VRS on CT images.

\section{ACKNOWLEDGMENTS}

This study was supported by a grant from the Korean Healthcare Technology R\&D Project, the Ministry for Health, Welfare \& Family Affairs, Republic of Korea (HI10C2020).

Authors' disclosures available online (http://www.jalz.com/disclosures/view.php?id=2109).

\section{SUPPLEMENTARY MATERIAL}

The supplementary table is available in the electronic version of this article: http://dx.doi.org/10.3233/ JAD-132333.

\section{REFERENCES}

[1] Denihan A, Wilson G, Cunningham C, Coakley D, Lawlor BA (2000) CT measurement of medial temporal lobe atrophy in Alzheimer's disease, vascular dementia, depression and paraphrenia. Int J Geriatr Psychiatry 15, 306-312.

[2] Jack CR, Jr., Petersen RC, Xu YC, Waring SC, O'Brien PC, Tangalos EG, Smith GE, Ivnik RJ, Kokmen E (1997) Medial temporal atrophy on MRI in normal aging and very mild Alzheimer's disease. Neurology 49, 786-794.

[3] Giesel FL, Hahn HK, Thomann PA, Widjaja E, Wignall E, von Tengg-Kobligk H, Pantel J, Griffiths PD, Peitgen HO, Schroder J, Essig M (2006) Temporal horn index and volume of medial temporal lobe atrophy using a new semiautomated method for rapid and precise assessment. AJNR Am J Neuroradiol 27, 1454-1458.

[4] Mizuno K, Wakai M, Takeda A, Sobue G (2000) Medial temporal atrophy and memory impairment in early stage of Alzheimer's disease: An MRI volumetric and memory assessment study. J Neurol Sci 173, 18-24.

[5] Launer LJ, Scheltens P, Lindeboom J, Barkhof F, Weinstein HC, Jonker C (1995) Medial temporal lobe atrophy in an open population of very old persons: Cognitive, brain atrophy, and sociomedical correlates. Neurology 45, 747-752.
[6] Barkhof F, Polvikoski TM, van Straaten EC, Kalaria RN, Sulkava R, Aronen HJ, Niinisto L, Rastas S, Oinas M, Scheltens P, Erkinjuntti T (2007) The significance of medial temporal lobe atrophy: A postmortem MRI study in the very old. Neurology 69, 1521-1527.

[7] Scheltens P, Leys D, Barkhof F, Huglo D, Weinstein HC, Vermersch P, Kuiper M, Steinling M, Wolters EC, Valk J (1992) Atrophy of medial temporal lobes on MRI in "probable" Alzheimer's disease and normal ageing: Diagnostic value and neuropsychological correlates. J Neurol Neurosurg Psychiatry 55, 967-972.

[8] Dickerson BC, Salat DH, Bates JF, Atiya M, Killiany RJ, Greve DN, Dale AM, Stern CE, Blacker D, Albert MS, Sperling RA (2004) Medial temporal lobe function and structure in mild cognitive impairment. Ann Neurol 56, 27-35.

[9] DeCarli C, Frisoni GB, Clark CM, Harvey D, Grundman M, Petersen RC, Thal LJ, Jin S, Jack CR, Jr., Scheltens P (2007) Qualitative estimates of medial temporal atrophy as a predictor of progression from mild cognitive impairment to dementia. Arch Neurol 64, 108-115.

[10] Korf ES, Wahlund LO, Visser PJ, Scheltens P (2004) Medial temporal lobe atrophy on MRI predicts dementia in patients with mild cognitive impairment. Neurology 63, 94-100.

[11] Rusinek H, Endo Y, De Santi S, Frid D, Tsui WH, Segal S, Convit A, de Leon MJ (2004) Atrophy rate in medial temporal lobe during progression of Alzheimer disease. Neurology $\mathbf{6 3}$, 2354-2359.

[12] Visser PJ, Verhey FR, Hofman PA, Scheltens P, Jolles J (2002) Medial temporal lobe atrophy predicts Alzheimer's disease in patients with minor cognitive impairment. JNeurol Neurosurg Psychiatry 72, 491-497.

[13] Jack CR, Jr., Albert MS, Knopman DS, McKhann GM, Sperling RA, Carrillo MC, Thies B, Phelps CH (2011) Introduction to the recommendations from the National Institute on Aging-Alzheimer's Association workgroups on diagnostic guidelines for Alzheimer's disease. Alzheimers Dement 7, 257-262.

[14] Wahlund LO, Julin P, Johansson SE, Scheltens P (2000) Visual rating and volumetry of the medial temporal lobe on magnetic resonance imaging in dementia: A comparative study. J Neurol Neurosurg Psychiatry 69, 630-635.

[15] Westman E, Cavallin L, Muehlboeck JS, Zhang Y, Mecocci P, Vellas B, Tsolaki M, Kloszewska I, Soininen H, Spenger C, Lovestone S, Simmons A, Wahlund LO, AddNeuroMed C (2011) Sensitivity and specificity of medial temporal lobe visual ratings and multivariate regional MRI classification in Alzheimer's disease. PLoS One 6, e22506.

[16] Kim GH, Kwon HJ, Go SA, Kim JE, Park KD, Choi KG, Im KH, Lee JM, Na DL, Jeong JH (2009) T1-axial medial temporal atrophy visual rating: A comparable study with schelten's t1-coronal visual rating. Dement Neurocognitive Disord 8, 37-44.

[17] McKhann G, Drachman D, Folstein M, Katzman R, Price D, Stadlan EM (1984) Clinical diagnosis of Alzheimer's disease: Report of the NINCDS-ADRDA Work Group under the auspices of Department of Health and Human Services Task Force on Alzheimer's Disease. Neurology 34, 939-944.

[18] Han C, Jo SA, Jo I, Kim E, Park MH, Kang Y (2008) An adaptation of the Korean mini-mental state examination (K-MMSE) in elderly Koreans: Demographic influence and population-based norms (the AGE study). Arch Gerontol Geriatr 47, 302-310.

[19] Ahn HJ, Chin J, Park A, Lee BH, Suh MK, Seo SW, Na DL (2010) Seoul Neuropsychological Screening Batterydementia version (SNSB-D): A useful tool for assessing 
and monitoring cognitive impairments in dementia patients. J Korean Med Sci 25, 1071-1076.

[20] Duvernoy HM (1988) The Human Hippocampus: An Atlas of Applied Anatomy. J.F. Bergmann, Munich.

[21] Mossman D, Somoza E (1991) ROC curves, test accuracy, and the description of diagnostic tests. J Neuropsychiatry Clin Neurosci 3, 330-333.

[22] Youden WJ (1950) Index for rating diagnostic tests. Cancer 3, 32-35.

[23] Schisterman EF, Perkins NJ, Liu A, Bondell H (2005) Optimal cut-point and its corresponding Youden Index to discriminate individuals using pooled blood samples. Epidemiology 16, 73-81.

[24] Li Y, Li J, Segal S, Wegiel J, De Santi S, Zhan J, de Leon MJ (2006) Hippocampal cerebrospinal fluid spaces on MR imaging: Relationship to aging and Alzheimer disease. AJNR Am J Neuroradiol 27, 912-918.

[25] de Leon MJ, Convit A, George AE, Golomb J, de Santi S, Tarshish C, Rusinek H, Bobinski M, Ince C, Miller D, Wisniewski H (1996) In vivo structural studies of the hippocampus in normal aging and in incipient Alzheimer's disease. Ann N Y Acad Sci 777, 1-13.

[26] Hyman BT, Van Hoesen GW, Damasio AR (1990) Memoryrelated neural systems in Alzheimer's disease: An anatomic study. Neurology 40, 1721-1730.

[27] Wattjes MP, Henneman WJ, van der Flier WM, de Vries O, Traber F, Geurts JJ, Scheltens P, Vrenken H, Barkhof F (2009) Diagnostic imaging of patients in a memory clinic: Comparison of MR imaging and 64-detector row CT. Radiology 253, 174-183. 